\title{
3D Feature-Scale Simulation of Sputter Etching with Coupling to Equipment Simulation
}

\author{
E. Bär, J. Lorenz, H. Ryssel \\ Fraunhofer Institute of Integrated Systems and Device Technology, \\ Schottkystrasse 10, 91058 Erlangen, Germany \\ eberhard.baer@iisb.fraunhofer.de
}

\begin{abstract}
Feature-scale simulation of sputter etching has been coupled to equipment parameters by means of transferring angular distributions of ions as provided by equipment simulation to our simulation code. Etching is modeled by performing ion flux integration for all node positions on a discretized 3D surface, taking into account shadowing by the geometry, the angular distribution of ions, and the sputtering yield. For rotationally symmetric geometries, a numerically and geometrically independent approach has been used to validate the $3 \mathrm{D}$ code. The application to $3 \mathrm{D}$ geometries is demonstrated for an L-shaped mask over a substrate for a resist mask geometry obtained from external lithography simulation.
\end{abstract}

\section{Introduction}

Accurate modeling of pattern transfer by lithography followed by dry etching becomes increasingly important for microelectronic fabrication as feature sizes shrink and the geometry of active and passive devices significantly impacts on performance and reliability. Predictive simulation of etching profile evolution requires coupling of reactor-scale simulation with feature-scale simulation. We present for the first time a triangle-based full 3D (not slice-wise 2D) etching simulator that allows implementation of physical-based surface models combined with the usage of data provided by equipment simulation, such as species concentrations or angular distributions of ions. Our program supports arbitrary 3D mask shapes as they can be obtained e.g. by 3D lithography simulation. In the following, our approach is demonstrated for sputter etching of silicon dioxide in an Ar plasma.

\section{Simulation Approach}

For sputter etching the etch rate is determined by the local flux of ions and the socalled sputter yield which is defined as the number of atoms sputtered per incident ion. For the examples below, we use experimental yield data [1] that describe the dependence on the angle between the velocity of the ion and the surface normal (Figure 1). The angular distribution of ions can be provided by equipment simulation or measurements. To determine the local etch rate for each triangle of the exposed oxide surface, numerical integration is carried out (over the free solid angle $\Omega_{\text {free }}$ ) of the product of the local ion flux and the sputter yield (both for the direction corresponding to $d \Omega$ ). The surface update is carried out in a sequence of steps in order to allow re-calculation of the local etch rates which vary with the changing geometry. 


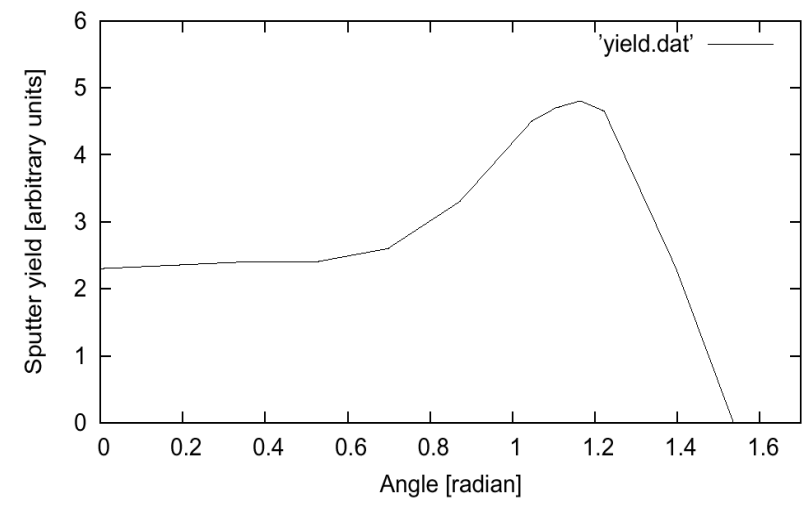

Figure 1: Yield function for sputter etching of $\mathrm{SiO}_{2}$ by $\mathrm{Ar}$ ions [1] as used for our simulations. The angle is taken between the ion velocity and the surface normal.

\section{Results}

Before studying a full 3D geometry we verify our numerical and geometrical engine by comparison with independent calculations. For contact hole etching with a cylindrical mask opening (width of opening is $w$, height of mask layer is $h$ ) the etched depth in the center of the contact hole can be evaluated for a given angular distribution $f(\vartheta)$ of the etching species and a given yield function $y(\vartheta)$ by:

$$
d_{\text {cent }}=\int_{0}^{d_{\text {field }}} d x\left[\frac{\int_{0}^{\arctan \left(\frac{w}{2(h+x)}\right)}[(\vartheta) y(\vartheta) \cos (\vartheta) d \vartheta}{\int_{0}^{\pi / 2} f(\vartheta) y(\vartheta) \cos (\vartheta) d \vartheta}\right]
$$

Here $d_{\text {cent }}$ and $d_{\text {field }}$ are the etched depth at the center of the contact hole and at unshadowed positions, respectively. In this context, the angular distribution $f(\vartheta)$ specifies the number of particles per interval $d \vartheta$. To obtain a distribution per solid angle interval $d \Omega, f(\vartheta)$ has to be divided by $\sin (\vartheta)$. For a thickness $d x$ etched in the field region, the etched thickness at the bottom center of the hole is obtained by multiplying $d x$ with the ratio of the integrated flux over the visible solid angle for this position (numerator of integrand) and the integrated flux for full view (denominator of integrand). The integrals have been solved numerically and compared to our 3D simulations which run completely independently from the numerical evaluation of eq. (1). We used ion angular distributions (IAD) of Ar that have been obtained by equipment simulation for varying process conditions [3]. Of particular importance for the IAD is the operating pressure. Increasing the pressure leads to a higher number of 
collisions within the sheath region. This increases the amount of ions with larger incident angles (Figure 2).

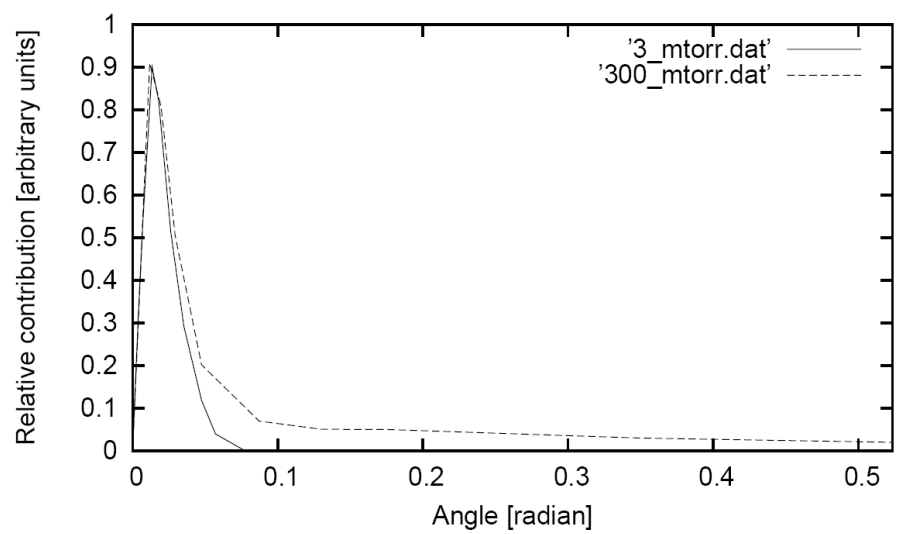

Figure 2: Angular distributions of $\mathrm{Ar}$ ions calculated by plasma equipment simulation [3]. Pressure $\mathrm{p}=3 \mathrm{mTorr}$ (continuous curve), $\mathrm{p}=300$ mTorr (dashed curve). The angle is taken between the direction of the ion velocity and the wafer normal.

In Figure 3, cross sectional views of the 3D simulations are shown for etching at 3 mTorr and $300 \mathrm{mTorr}$, with $d_{\text {field }}=0.5 \mu \mathrm{m}$. The results for the two pressures differ significantly. In particular, the etched depths are largely different, i.e. $d_{\text {center }}(3 \mathrm{mTorr})=0.5 \mu \mathrm{m}$ and $d_{\text {center }}(300 \mathrm{mTorr})=0.3561 \mu \mathrm{m}$. The values predicted by the independent calculations according to Eq. (1) are $0.5 \mu \mathrm{m}$ and $0.3553 \mu \mathrm{m}$, respectively, which agree well with our 3D simulation. Possible reasons for the remaining difference are the finite triangles size for surface discretization, the finite number of surface update steps for the simulation run, and the deviation of the discretized 3D geometry from perfect rotational symmetry.
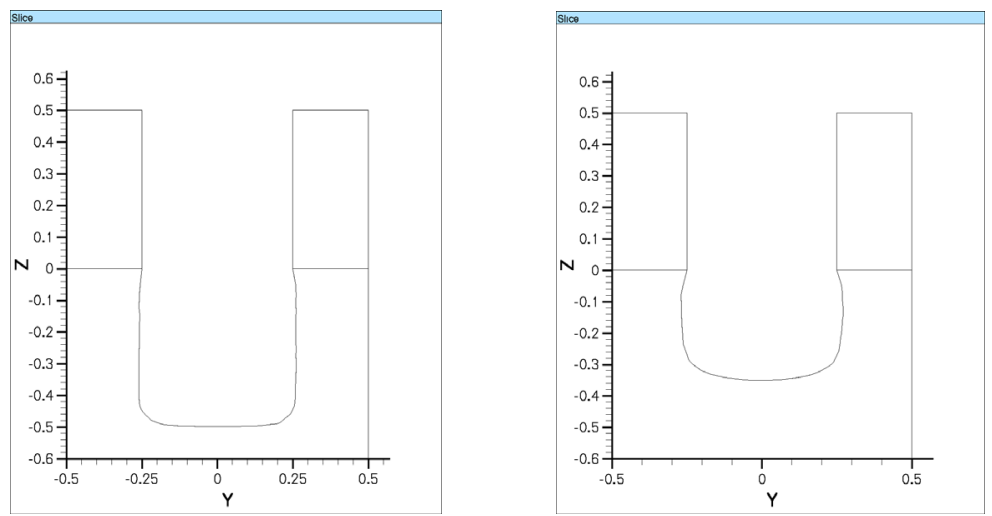

Figure 3: Simulation of contact hole etching for 3 mTorr (left) and $300 \mathrm{mTorr}$ (right).

Now we consider an L-shaped trench which requires 3D treatment both in terms of particle transport modeling and surface representation. The initial geometry (Figure 4, left) has been obtained by lithography simulation (with exposure at $248 \mathrm{~nm}$ 
wavelength) using SOLID-CTM [2]. Figure 4 (right) shows the simulation results for etching at $3 \mathrm{mTorr}$ and $300 \mathrm{mTorr}$. In both cases, an etched depth of $0.4 \mu \mathrm{m}$ for unshadowed positions has been specified. The underetching is more pronounced for the higher pressure which is due to the relatively higher number of ions with larger incident angles. Also the bottom shapes of the etched trenches differ due to the different IADs. For the IAD at 300 mTorr shadowing caused by the trench mask has a stronger effect than for the narrower distribution at $3 \mathrm{mTorr}$, thus leading to a reduced etch depth for the higher pressure.
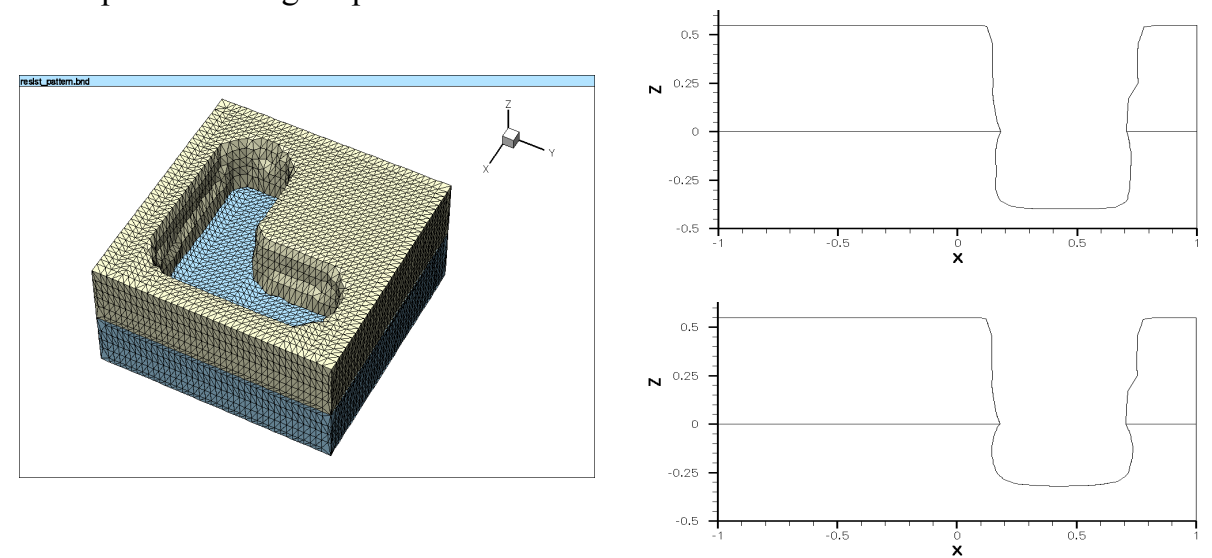

Figure 4: Resist pattern over oxide layer before (left) and after (cross sections, right) etching simulation for 3 mTorr (top right) and $300 \mathrm{mTorr}$ (bottom right). Cross sections through the center of the simulation region and perpendicular to the y-axis.

\section{Conclusions}

The link between profile evolution and equipment parameters has been demonstrated by coupling feature-scale simulation of sputter etching with IAD data obtained from equipment simulation. The module is currently being extended to allow modeling of further processes such as reactive ion etching. This requires additional information to be transferred from equipment simulation (such as concentrations of different species) and the usage of refined surface models describing the complex interaction of neutral and ionic species involved in the etching process.

Acknowledgement

This work has been funded in part by the IST project 2000-30133 MULSIC.

\section{References}

[1] J.P. Chang, H.H. Sawin, Molecular-Beam Study of the Plasma-Surface Kinetics of Silicon Dioxide and Photoresist Etching with Chlorine, J. Vac. Sci. Technol. 19 (2001) 1319.

[2] Lithography Simulator SOLID-CTM, Release 6.3.0, SIGMA-C GmbH, Munich, 2003.

[3] M. Kratzer, R.P. Brinkmann, W. Sabisch, H. Schmidt, Hybrid Model for the Calculation of Ion Distribution Functions behind a Direct Current or Radio Frequency Driven Plasma Boundary Sheath, J. Appl. Phys. 90 (2001) 2169. 